Proc. 13th International School on Theoretical Physics: Symmetry and Structural Properties of Condensed Matter

\title{
Magnetic Anisotropy in Doped Graphene with Rashba Spin-Orbit Interaction
}

\author{
M. InGLOT* AND V.K. DUGAEV \\ Department of Physics and Medical Engineering, Rzeszów University of Technology, \\ al. Powstańców Warszawy 6, 35-959 Rzeszów, Poland
}

\begin{abstract}
We present our calculations of on-site magnetic anisotropy (MA) in graphene with magnetic atoms. We assume that the graphene layer is placed on a substrate, which generates the homogeneous Rashba spin-orbit field. The resonant state in the presence of Rashba spin-orbit interaction is calculated for two possible orientations of the magnetic moment namely perpendicular, $S_{z}=1$, and parallel, $S_{z}=0$, to the graphene plane. The magnetic anisotropy is calculated as a difference between the energies of these states and presented as a function of impurity potential $g_{0}$ and magnitude of the Rashba spin-orbit coupling $\alpha$.
\end{abstract}

DOI: 10.12693/APhysPolA.135.1249

PACS/topics: graphene, magnetic anisotropy, Rashba spin-orbit interaction, magnetic impurity

\section{Introduction}

Graphene is a single layer of carbon atoms with the gapless electronic structure $[1,2]$. The natural spin-orbit interaction (SOI) in graphene, usually called intrinsic SOI, can modify the low-energy spectrum leading to the energy gap (around $\sim \mu \mathrm{eV}$ ) [3] but the magnitude of this interaction is so small that it can be completely neglected in calculations. Another way to change the electron structure of graphene is to use a substrate breaking the crystal symmetry in 2D system [4]. This type of spin-orbit interaction plays crucial role in graphene applications in spintronics [5].

Magnetic properties of carbon-based materials are of great interest for physics and applications. One of the possible ways to change the magnetic properties in graphene is related to the doping with impurities. The disorder in doped graphene can be realized with a longor short-range potential profile with random or ordered distribution of impurities [6-8].

The problem of magnetic anisotropy in graphene attracted a lot of interest recently. In particular, high magnetic anisotropy has been predicted for graphene decorated with transition-metal adatoms and dimers [9]. By using the density functional theory (DFT) method, the magnetic anisotropy parameter was calculated in graphene quantum dots [10]. Recently, the magnetic anisotropy has been observed in single or bilayer graphene [11], graphene oxides [12], and in graphene/magnetic heterostructures [13].

The existence of resonance states in semiconductors leads to strong changes of their transport properties. This problem has been considered for single and bilayer

\footnotetext{
* corresponding author
}

graphene $[14,15]$. Recently, the problem of resonant scattering affecting the momentum relaxation time of electrons in graphene has been investigated by Irmer et al. (see Ref. [16]).

In this paper we consider the implementation of magnetic atoms with short-range potential $\hat{V}_{0}$ located in one of graphene sublattices, A or B [17-19]. Such an impurity can generate local magnetic moment by electron occupation of the resonant state $[20,21]$. We consider the case of rather low concentration of magnetic impurities so that the effects of their interaction can be neglected.

\section{Model}

To calculate the electron energy spectrum of graphene on substrate we use the relativistic model described by the Dirac Hamiltonian of massless electrons with the Rashba spin-orbit interaction

$$
\hat{H}_{k}=v\left( \pm \tau_{x} k_{x}+\nu \tau_{y} k_{y}\right)+\lambda\left( \pm \tau_{x} \sigma_{y}-\tau_{y} \sigma_{x}\right),
$$

where $v=\hbar v_{F}=\hbar c / 300, c$ is the speed of light, the matrices $\boldsymbol{\tau}=\left(\tau_{x}, \tau_{y}\right)$ and $\boldsymbol{\sigma}=\left(\sigma_{x}, \sigma_{y}\right)$ are the Pauli matrices acting in sublattice (A or $\mathrm{B}$ ) and spin spaces, respectively. The sign \pm refer to nonequivalent Dirac points $K$ and $K^{\prime}$ in the Brillouin zone. The eigenvalues of the Hamiltonian (1) are $E=\mp \varepsilon_{k}$, where $\varepsilon_{k}=\lambda \pm \sqrt{v^{2} k^{2}+\lambda^{2}}$.

The impurity state associated with a single magnetic atom localized in one of the sublattices in graphene can be calculated with the perturbation localized at $\boldsymbol{r}=0$ :

$$
\hat{V}(\boldsymbol{r})=g \delta(\boldsymbol{r})\left(\begin{array}{cc}
\boldsymbol{S} \cdot \boldsymbol{\sigma} & 0 \\
0 & 0
\end{array}\right),
$$

where $g$ is the coupling constant and the vector of magnetic moment $\boldsymbol{S}$ has an arbitrary orientation. The matrix of perturbation in the basis functions of Hamiltonian (1) has the following form:

$$
\hat{V}_{\boldsymbol{k} \boldsymbol{k}^{\prime}}=\frac{g}{2}\left(1+\tau_{z}\right) \boldsymbol{S} \cdot \boldsymbol{\sigma} \equiv \hat{V}_{0} .
$$


In order to find the energy of localized state of doped graphene with the Rashba spin-orbit interaction we use the $T$-matrix method, in which the equation for $T$-matrix is

$$
\hat{T}_{\boldsymbol{k} \boldsymbol{k}^{\prime}}(\varepsilon)=\hat{V}_{\boldsymbol{k} \boldsymbol{k}^{\prime}}+\sum_{\boldsymbol{k}_{1}} \hat{V}_{\boldsymbol{k} \boldsymbol{k}_{1}} \hat{G}_{\boldsymbol{k}_{1}}(\varepsilon) \hat{T}_{\boldsymbol{k}_{1} \boldsymbol{k}}(\varepsilon),
$$

and the Green function of Hamiltonian (1), $\hat{G}_{\boldsymbol{k}}(\varepsilon)=$ $\left(\varepsilon-\hat{H}_{\boldsymbol{k}}\right)^{-1}$, can be written as

$$
\hat{G}_{k}(\varepsilon)=\frac{\varepsilon+v \boldsymbol{\tau} \cdot \boldsymbol{k}+\lambda\left(\tau_{x} \sigma_{y}-\tau_{y} \sigma_{x}\right)}{\varepsilon^{2}-\varepsilon_{k}^{2}} .
$$

Equation (4) means that we account for perturbation (2) in all orders of magnitude.

We assume that the energy parameter $\varepsilon$ includes a small imaginary part $\varepsilon \rightarrow \varepsilon+\mathrm{i} \delta$, which corresponds to the retarded Green function. Using Eqs. (4)-(5) we find

$$
\hat{T}(\varepsilon)=\left[1-\hat{V}_{0} \hat{F}(\varepsilon)\right]^{-1} \hat{V}_{0},
$$
where $\hat{F}(\varepsilon)=\sum_{\boldsymbol{k}} \hat{G}_{\boldsymbol{k}}(\varepsilon)$. The resonant states can be found from the equation, defining the poles of $T$-matrix

$$
\operatorname{det}\left[\left(1-\hat{V}_{0} \hat{F}(\varepsilon)\right)^{-1} \hat{V}_{0}\right]=0 \text {. }
$$

After calculating the determinant we can present Eq. (7) in the following form:

$$
1-g_{0}^{2} \vartheta_{1} \vartheta_{2}+g_{0} S_{z}\left(\vartheta_{1}-\vartheta_{2}\right)=0
$$

where

$$
\begin{aligned}
& \vartheta_{1}=\sum_{\boldsymbol{k}} \frac{k \varepsilon\left(\varepsilon^{2}-v^{2} k^{2}\right)}{\varepsilon^{4}-4 \lambda^{2} \varepsilon^{2}-2 v^{2} k^{2} \varepsilon^{2}+v^{4} k^{4}}, \\
& \vartheta_{2}=\sum_{\boldsymbol{k}} \frac{k \varepsilon\left(\varepsilon^{2}-v^{2} k^{2}-4 \lambda^{2}\right)}{\varepsilon^{4}-4 \lambda^{2} \varepsilon^{2}-2 v^{2} k^{2} \varepsilon^{2}+v^{4} k^{4}} .
\end{aligned}
$$

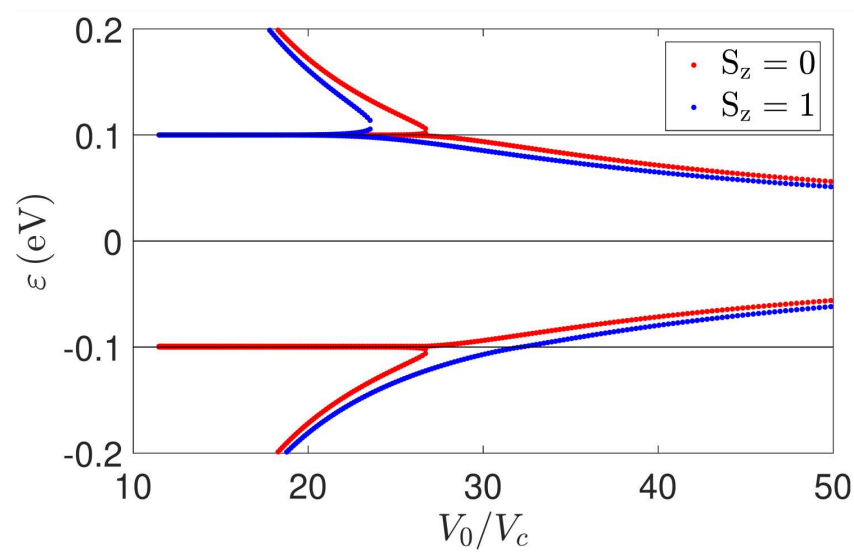

Fig. 1. Energy of localized state in graphene with the Rashba spin-orbit interaction $2 \lambda=\alpha=100 \mathrm{meV}$ as a function of $g_{0} / g_{c}>0$, where $g_{c}=a_{0}^{2} \varepsilon_{c}$ for two types of magnetic orientation. Red colour - magnetic moment is parallel to the graphene plane, $S_{z}=0$, and blue colour - magnetic moment is perpendicular to the plane, $S_{z}=1$.

Using Eq. (8) we calculate the energy of resonant states $\varepsilon$ for different angles of impurity magnetic moment $\mathbf{S}$. The dependence of this energy on $g_{0}$ is presented in Fig. 1, where the blue color corresponds to spin perpendicular to the graphene plane while the red corresponds to the in-plane spin.

We define the MA as a difference between the energy of localized states $\mathcal{E}=\left|\varepsilon_{\uparrow}-\varepsilon_{0}\right|$ for given potential $g_{0}$. In our calculation we use the Rashba spin-orbit coupling equal to $\alpha=100 \mathrm{meV}[22-24]$. The value of MA as a function of energy $\bar{E}=\left|\varepsilon_{\uparrow}-\varepsilon_{0}\right| / 2$ is presented in Fig. 2a. In Fig. $2 \mathrm{~b}$ for $\varepsilon>0$ and $\varepsilon<0$ we can see two regions of the dependence of MA. The first one is for small impurity potential $\sim 25 g_{0} / g_{c}$, where the MA can have two different values. The magnetic anisotropy for impurity potential larger than $25 g_{0} / g_{c}$ is weak and depends very weakly on $g_{0}$.
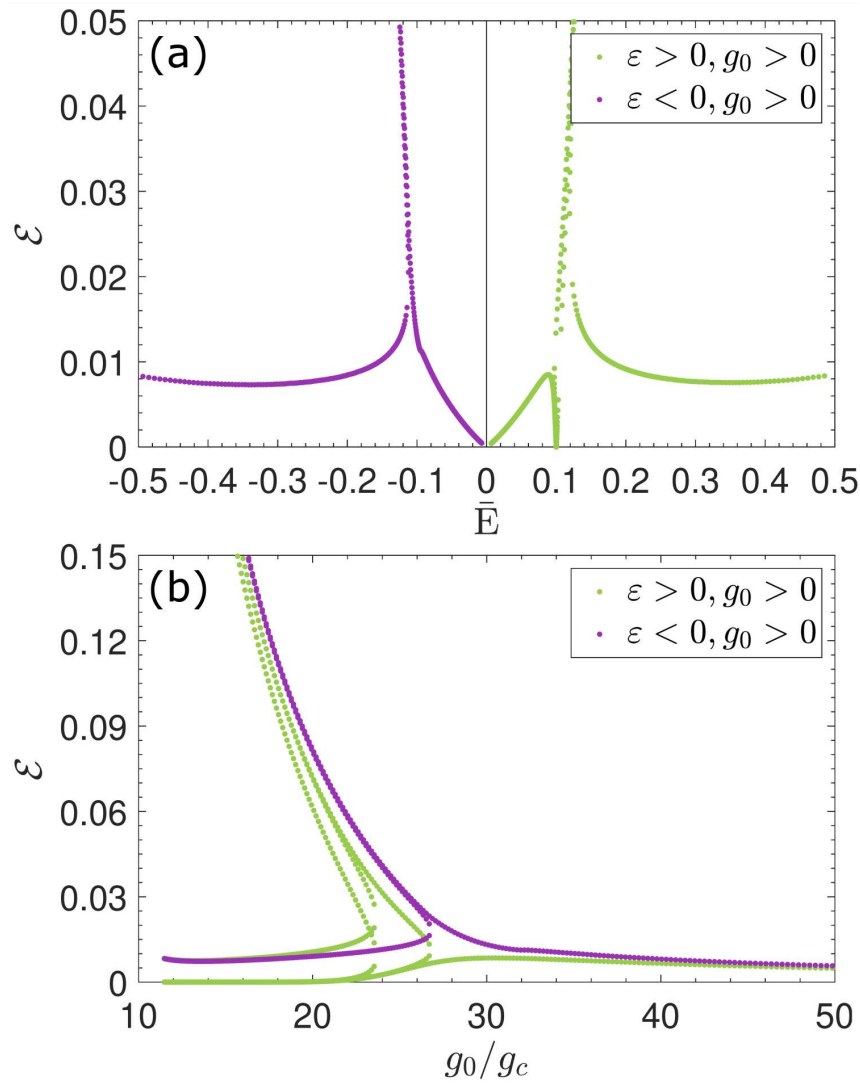

Fig. 2. Magnetic anisotropy (a) as function of average value of energy for impurity potential $g_{0} / g_{c}=11$, (b) as a function of impurity potential $g_{0}$. In both figures Rashba spin-orbit coupling is $\alpha=100 \mathrm{meV}$.

The energy level of localized state depends on the strength of the Rashba spin-orbit coupling (see Fig. 1). The magnitude of coupling constant can by modified by external electric field. The possibility of controlling Rashba spin-orbit coupling makes it possible to control the magnetic properties doped graphene. From Fig. 3a and $b$ we see that in order to reach observable magnetic anisotropy one can use magnetically doped graphene on a substrate with relatively weak Rashba spin-orbit coupling. 

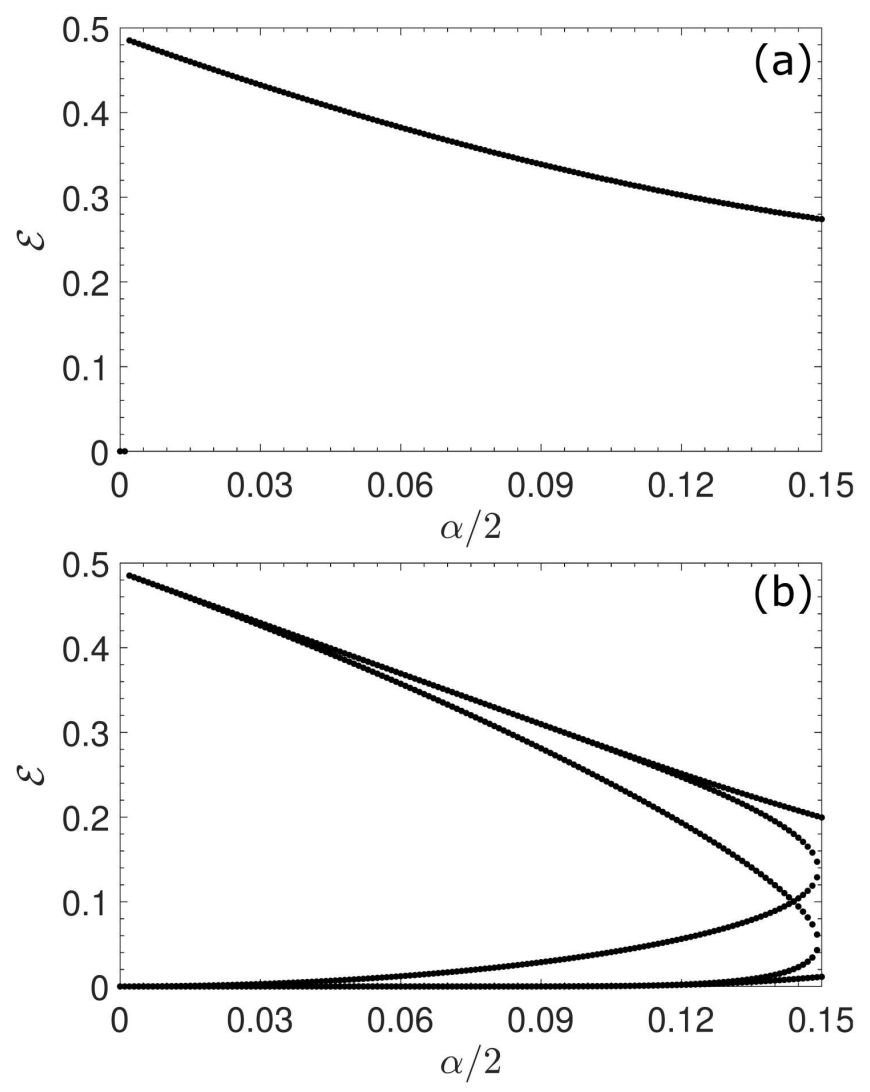

Fig. 3. (a) Maximum value of magnetic anisotropy as a function of Rashba spin orbit coupling $\alpha$ in the width range of impurity potential $g_{0} / g_{c}=[10,300]$. (b) Magnetic anisotropy as a function of Rashba spin-orbit coupling $\alpha$ for chosen impurity potential $g_{0} / g_{c}=11$.

\section{Conclusion}

We calculated the energy of a resonant level created by magnetic impurity in graphene with the Rashba spinorbit interaction. Our main result is that this energy is dependent on the orientation of magnetic moment of impurity with respect to the graphene plane. This leads to appearance of on-site magnetic anisotropy provided that the corresponding impurity level is occupied by electron. As we see from Fig. 1, the energy of localized state is lower if the moment is perpendicular to the plane. Thus, the off-plane orientation of the moment is energetically favorable.

Our results also demonstrate that one can control the structure of electron energy states by using the reorientation of magnetic moments of impurities on graphene. This can affect electrical properties of graphene, and the effect can be rather strong due to the resonant scattering of electrons from magnetic impurities.

\section{Acknowledgments}

This work is supported by the National Science Center in Poland as a research project No. DEC$2017 / 27 /$ B/ST3/02881.

\section{References}

[1] K.S. Novoselov, Science 306, 666 (2004).

[2] K.S. Novoselov, Nature 438, 197 (2005).

[3] Y. Yao, F. Ye, X.L. Qi, S.C. Zhang, Z. Fang, Phys. Rev. B 75, 041401 (2007).

[4] C.L. Kane, E.J. Mele, Phys. Rev. Lett. 95, 226801 (2005).

[5] H. Min, J.E. Hill, N.A. Sinitsyn, B.R. Sahu, L. Kleinman, A.H. MacDonald, Phys. Rev. B 74, 165310 (2006).

[6] Y.V. Skrypnyk, V.M. Loktev, Phys. Rev. B 73 241402 (2006).

[7] V.M. Pereira, J.M.B. Lopes dos Santos, A.H. Castro Neto, Phys. Rev. B 77, 115109 (2008).

[8] D.M. Basko, Phys. Rev. B 78, 115432 (2008).

[9] L.Z. Zhang, Z.F. Wang, S.X. Du, H.J. Gao, F. Liu, Phys. Rev. B 90, 161402 (2014).

[10] I. Beljakov, V. Meded, F. Symalla, K. Fink, S. Shallcross, W. Wenzel, B.J., Nanotechnol. 4, 441 (2013)

[11] M. Temmen, O. Ochedowski, B.K. Bussmann, M. Schleberger, M. Reichling, T.R.J. Bollmann, Nanotechnology 4, 625 (2013)

[12] K. Ning, H. Liu, L. Li, H. Li, J. Feng, B. Yang, X. Liu, Y. Li, Y. Chen, H. Wei, X. Han, S. Mao, X. Zhang, Y. Yang, T.L. Ren, RSC Adv. 7, 52938 (2017).

[13] H. Yang, A.D. Vu, A. Hallal, N. Rougemaille, J. Coraux, G. Chen, A.K. Schmid, M. Chshiev, Nano Lett. 16, 145 (2016).

[14] A. Ferreira, J. Viana-Gomes, J. Nilsson, E.R. Mucciolo, N.M.R. Peres, A.H. Castro Neto, Phys. Rev. B 83, 165402 (2011).

[15] M. Monteverde, C. Ojeda-Aristizabal, R. Weil, K. Bennaceur, M. Ferrier, S. Guéron, C. Glattli, H. Bouchiat, J.N. Fuchs, D.L. Maslov, Phys. Rev. Lett. 104, 126801 (2010).

[16] S. Irmer, D. Kochan, J. Lee, J. Fabian, Phys. Rev. B 97, 075417 (2018).

[17] M. Inglot, V.K. Dugaev, J. Appl. Phys. 109, 123709 (2011).

[18] I. Kupčić, Phys. Rev. B 90, 205426 (2014).

[19] M. Zhong, S. Li, H.J. Duan, L.B. Hu, M. Yang, R.Q. Wang, Sci. Rep. 7, 3971 (2017).

[20] P. Venezuela, R.B. Muniz, A.T. Costa, D.M. Edwards, S.R. Power, M.S. Ferreira, Phys. Rev. B 80, 241413 (2009).

[21] Y. Li, J. He, X. Kong, S.P. Kou, Phys. Rev. B 90, 201406 (2014).

[22] P. Leicht, J. Tesch, S. Bouvron, F. Blumenschein, P. Erler, L. Gragnaniello, M. Fonin, Phys. Rev. B 90, 241406 (2014).

[23] Y.S. Dedkov, M. Fonin, U. Rüdiger, C. Laubschat, Phys. Rev. Lett. 100, 107602 (2008).

[24] E.I. Rashba, Phys. Rev. B 79, 161409 (2009). 